\title{
MODERN METHODS OF DIAGNOSING VIRTUAL ADDICTION AMONG ADOLESCENTS: RELEVANCE AND PROSPECTS
}

\section{Serhii Maksymenko ${ }^{1}$ \\ Oleksii Lysenko ${ }^{2}$}

DOI: https://doi.org/10.30525/978-9934-588-15-0-124

\begin{abstract}
Modern development of media technologies helps users of all ages get effective access to information of interest. A variety of Internet resources, video games, social networks, sites with goods allows a person to delve into the sphere of entertainment and hobbies. However, against the background of stress disorders, life difficulties, physical health and features of the psycho emotional sphere, the user of the virtual space may develop dependence on different media and Internet resources. According to the results of various studies, it was found that the special risk category is the population of adolescence. Therefore, the subject of our study is the study of the important problem of the optimal diagnosis of virtual dependence in adolescents. Diagnosis of virtual addiction among adolescents is based mainly on the study of the prevalence of interest in virtual space over vital interests, daily activities, household work, live communication with friends and relatives (without the use of media devices and an Internet connection during free time), etc. The list of diagnostic criteria is very wide and characterized by various nuances of psycho-emotional detection; so many questionnaires are constantly being improved. The purpose of this study is to determine international experience regarding the solution of the complex problem of diagnosing virtual addiction among adolescents, which occurs using various psychological questionnaires and instrumental methods. A large number of modified questionnaires take into account various factors: age, gender, social status, concomitant psychological disorders, physical health, type of virtual addiction, etc. The cause of the development of the disorder can be both biological factors and factors of psychological
\end{abstract}

\footnotetext{
${ }^{1}$ Professor, Director of the G.S. Kostiuk Institute of Psychology of the National Academy of Educational Sciences of Ukraine, Ukraine

${ }^{2}$ Graduate Student of the G.S. Kostiuk Institute of Psychology of the

National Academy of Educational Sciences of Ukraine, Ukraine
} 
perception. An important role may be in the individual characteristics of decision-making by a person, his emotional control, cognitive regulation, which, according to various literature data, can give in to additional vulnerabilities among adolescents due to the formation of hormonal surges, a tendency to make rash decisions, and falling under the destructive influence of other personalities. It is necessary to take into account the quality of the research, the presence of control groups, the number of participants, the methodology and other important factors that may affect the results. Materials and research methods are foreign publications that relate to the topic of diagnosing virtual dependence. In the process of the study, the authors of the article used a systematic, statistical and comparative analysis, compilation of scientific literature, which examined data that are freely available among the scientific databases of the world. Conclusions: the relevance and prospects of further research in this area are summarized on the basis of data on modern methods of diagnosing virtual dependence among adolescents. Nowadays psychological, as well as instrumental methods for determining this disorder are very diverse, have a variable degree of efficiency, accuracy and productivity depending on the research process. It is necessary to take further steps in the study of this problem.

\section{Introduction}

Humanity began to actively use virtual space about 20 years ago. However Internet and media devices usage has increased in recent years. Different country leaders are starting to introduce healthy Internet use programs at the legislative level. This is due to the problem of the development of suicidal activity, fraud, working degradation, psycho-physical disorders due to excessive or pathological enthusiasm for virtual space (see Figure 1).

It's necessary to understand the terminology and classification of this disorder for the high-quality development of legislative documents. That's why there are large numbers of modified questionnaires in the world, which appeared as a result of attempts to study this problem more accurately. Unfortunately, the variety of psychological methods for diagnosing virtual addiction can not only help, but also complicate the qualitative determination of the diagnosis due to the lack of accurate measures. The novelty of the work is that the article presented the latest data of the scientific literature concerning the study and diagnosis of virtual addiction in the context 


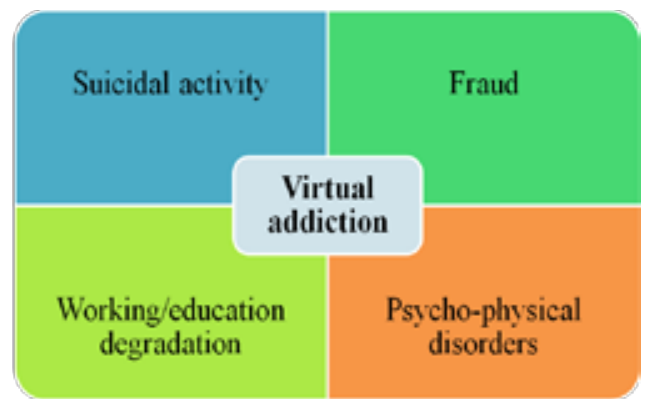

Figure 1. The effects of virtual addiction

of modern psychology. In comparison with chemical types of addictions (such as alcohol, tobacco addiction and substance abuse, etc.), where some of them have been known in medicine for many centuries, virtual addiction has appeared relatively recently. Despite the fact that the mechanisms of dependency have common features, researchers are faced with new problems that require a differential approach. Therefore, the treatment acquires multifactorial characteristics of the impact on the patient. Virtual addiction has different modifications of profiles, that's why, a differential approach to diagnosis and treatment is required. There are various errors during the study of the problem (for example, the lack of a fair number of respondents, control groups, drawbacks of forming questions that are included in diagnostic questionnaires, etc.). Working purpose is to study the relevance and prospects of further research on modern methods of diagnosing virtual addiction among adolescents. Optimal and scientifically based materials and research methods were used to obtain information on this topic. Mainly foreign publications, which relate to the topic of diagnosing virtual dependence, were studied in this work. Also, the authors of the article used systemic, statistical, and comparative methods for analyzing the received information. The authors of this article formed a methodology using the indicated types of analysis, studied the structure of the issue of virtual dependence diagnosis among adolescents. Research tools summarized the data of the scientific literature that were freely available among different scientific and metric databases of the world. Knowledge was formed about the interconnected elements of the study of this disorder. 


\section{The danger of virtual addiction for adolescents}

Easy access to information technology is associated with the growth of excessive / pathological use of the Internet and media devices. Round 1996, virtual dependence began to be designated as a global problem of various social stratum of the population. The virtual environment carries multifactorial risks for each age group, however, the biggest risk is typical for children and adolescents. Except internet scam, there are other online dangers that include cyber suicidal, self-harming behavior, sexual abuse, racial discrimination, intimidation with caused psycho-physical injuries and other negative consequences, therefore, effective programs for the prevention and treatment of virtual addiction are possible by using an interdisciplinary approach. It is necessary to make a detailed review of the existing symptoms of virtual dependence to form optimal diagnostic questionnaires for different age groups, including adolescents [7, p. 260-272]. According to this information and the latest statistics for 2016 provided by the World Health Organization (WHO), suicide ranked second on the causes of death among young people aged 15-29. Therefore, virtual addiction can

be a potential threat for this age category [14]. Over the past 45 years there has been a tendency to a critical increase of suicides, which is $60 \%$. The number should be even higher, but unsuccessful suicide attempts are not counted. The issue of suicidal activity is included in the WHO Program of Action for filling mental health gaps (mhGAP) [13].

\section{Research results}

\subsection{What is virtual addiction?}

Based on various data on the terminology of virtual dependence (cyberaddiction), the authors of the article developed a generalized definition of this disorder. Virtual addiction is a psychological disorder that occurs as a result of excessive and systematic use of media devices, which is manifested by gambling, addiction to social networks, web shopping, web search, watching pornographic materials, etc. This leads to a negative impact on a person's daily life.

\subsection{The relevance of the development of diagnostic questionnaires}

Virtual addiction has various types, which can manifest themselves differently depending on gender, age, social category, etc., it can have their 
own behavioral features. Unfortunately, the development of virtual addiction can complicate private communication and intimacy. It is important to choose the optimal research design (see Figure 2).

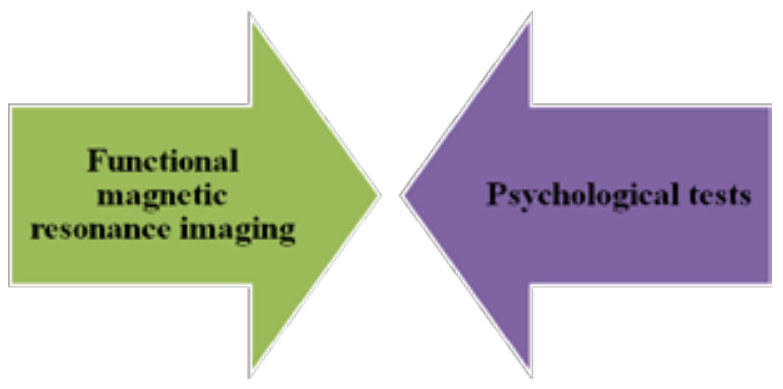

Figure 2. Types of diagnostics

It is important to pay attention to the need to use modified questionnaires or apply several types of testing, because in this article, we study such a target population as adolescents. This is due to the need to provide a higher level of the veracity of a results. The use of various approaches and questionnaires can significantly improve the quality of diagnosis, which is especially important in the conditions of modern life of a young person, where other related psycho-physical disorders are possible. After all, it is known that, by development of virtual addiction among adolescents, there are many mental disorders, organic lesions of the central nervous system, and other addictions, for example, alcohol, drugs and smoking, which can lead to distortion of the results. Research requires a solid approach and a very thoughtful analysis of the problem. By diagnostics and treatment virtual addiction, there are comorbide disorders (depression, anxiety disorders, hyperkinetic syndrome, etc.) to consider. And if it's necessary, doctor or psychologue can recommend a teenager not to use any digital devices that are addictive [15, p. 321-333]. Regardless of the officially established diagnosis of virtual addiction and its specific manifestations, people need highly qualified and professional help to solve personal problems. There are also psychiatric centers in some countries that focus on treating virtual games addiction and other Internet addictions. There are various methods of treatment, including medication. For example, psychotropic medications 
are being studied to treat cyberaddiction. According to publications for the virtual addiction treatment, there are medications based on bupropion, methylphenidate, etc. [18, p. 979-994].

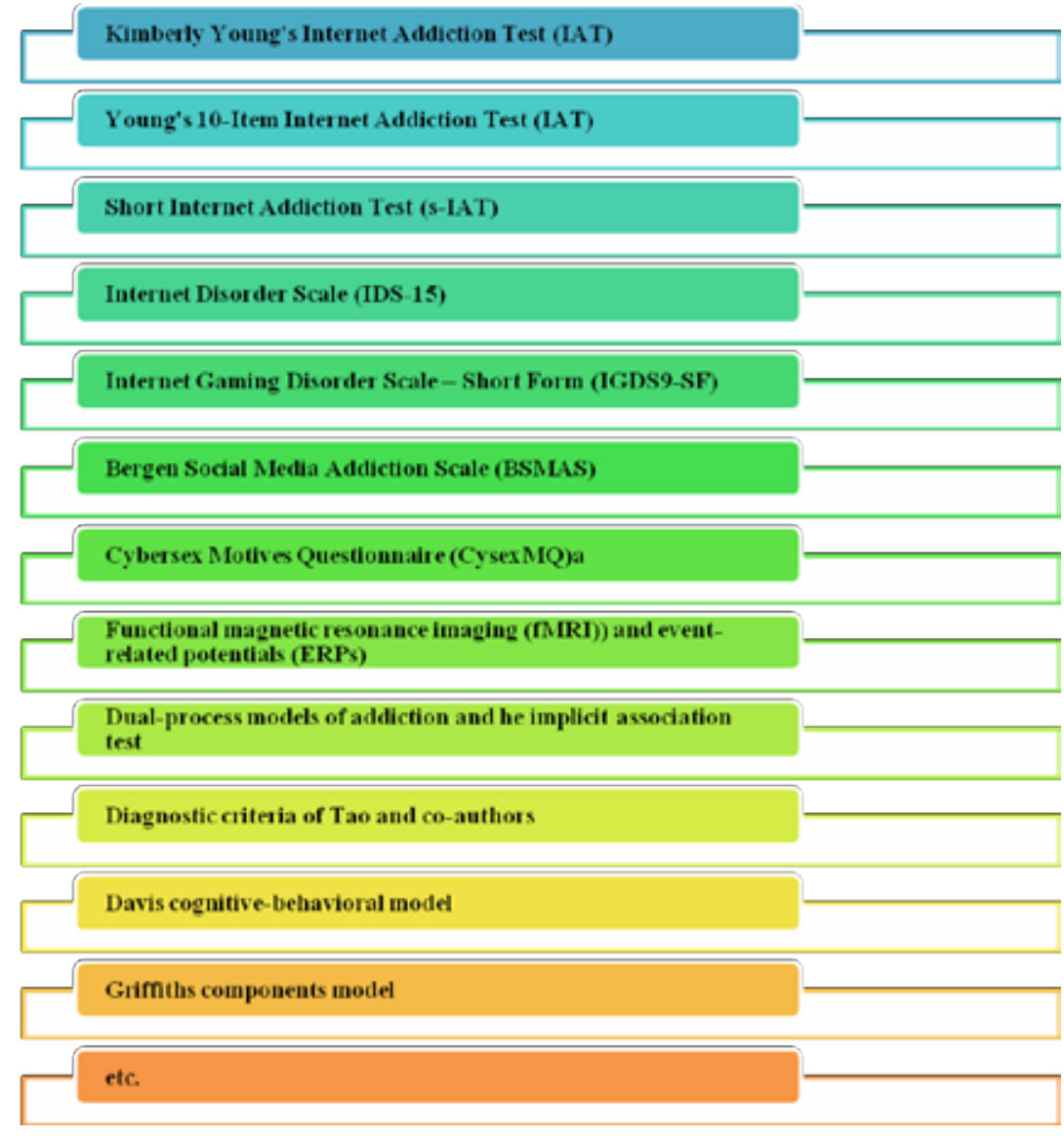

Figure 3. Modern diagnostic methods

\subsection{Kimberly Young's Internet Addiction Test (IAT)}

Kimberly Young first presented her research on Internet addiction in 1996 at the annual conference of the American Psychological Association. 
Many questionnaires have been published about this issue [6, p. 388-433]. Young used the established criteria in her work to determine gambling addiction to the game, so the questionnaire defines Internet addiction as a control disorder regarding personal impulsivity (where chemicals are not used). There are a set of criteria that include:

- Internet addiction;

- the need to use the Internet the great deal of time, as well as spending online more time as it planned;

- failed attempt to stop using the Internet;

- mood changes by trying to reduce or stop using it;

- risk of losing important relationships or opportunities because of Internet overuse;

- cheating about using the Internet;

- internet usage to avoid problems or desire to get rid of a bad mood;

Only personal (non-work or study) use of the Internet should be evaluated. The diagnosis of Internet addiction was established by 5 or more criteria. Conceptualization Young was popularized due to the extended 20-position test, suggested in 1998.

6 main diagnostic factors were obtained by the development of the questionnaire:

- Internet overuse;

- Expressivity;

- work / study neglect;

- neglect of real social life;

- anticipation of Internet usage;

- lack of self-control.

Many authors have noted that the questionnaire is a reliable assessment tool of the dependence level, mostly from the internet [16, p. 203-213]. Estimation is 100 -point scale. Low level - about $20-49$ points, moderate 50-79 points, serious - 80-100 points [17, e107306]. This type of questionnaire is often used to study virtual addiction.

2014 (Masaki, Ehime Prefecture, Japan) there was a test in which adolescents 12-15 years old in the amount of 853 people took part. Booking attendee list -425 men and 428 women. Young Internet Addiction Test (IAT) and the Japanese version of the General Health Questionnaire (GHQ) were used. Participants were asked to fill out a questionnaire regarding 
influence or access to electronic devices such as a phone, smartphone, laptop, etc. Respondents were interviewed about how exactly they use media devices and answered "yes" or "no" to the question about the degree of free access to equipment. The duration of utilization of each electronic device was also taken into account. Based on the proposed 20 IAT points, estimates are used from 1 to 5 (with values from 20 to 100). A large number of points is a tendency to addiction. Respondents with IAT scores above 70 points were classified as "addict". IAT ratings from 40 to 69 are "possible addiction", below 39 - "no addiction". The Cronbach coefficient (index, which is used to verify the reliability of the test results in psychology) was 0.929 . It was used GHQ (which consists of 30 points) to determine the mental state of adolescents (this is an approved version of the questionnaire in Japan, which is used to define adolescents well-being). GHQ was used to determine overall incidence, social dysfunction, somatic symptoms, sleep disturbance, anxiety, dysphoria, suicidal behavior, where the Cronbach coefficient was 0.915. Research results showed that smartphones usage (including mobile phones), laptops and game consoles correlated with Internet addiction, where the free availability of smartphones was the most significant factor [4, p. 405-412].

\subsection{Young's 10-Item Internet Addiction Test (IAT)}

There is a version of the questionnaire with 10 questions. It contains the following questions:

- the feeling of Internet or other online services addiction and thinking about it offline;

- the feeling of spending more and more time online to achieve satisfaction;

- vain endeavours to control online time usage;

- the feeling of anxiety or annoyance while trying to reduce or stop being online;

- spending online more time as it planned;

- risk of losing important relationships, work or study opportunities because of Internet overuse;

- cheating family or friends about Internet overuse;

- being online to avoid problems or reduce feelings of helplessness, guilt, anxiety or depression; 
- having depression, moodiness or irritability;

- using Internet even after paying too much money on it.

Respondents answered "Yes" or "No". The results should be identified on the basis of input data [11, p. 1-9].

\subsection{Short Internet Addiction Test (s-IAT)}

There is another German version of the Internet addiction test, which is based on the original version of the Young questionnaire, in which you need to answer 12 questions. There is five -point scale from 1 (= never) to 5 (= very often), so the total score ranges from 12 to 60 (> 30 mean problems with the use of the Internet, and rating $>37-$ pathology). The questionnaire determines the level of violation of time management in everyday life as a result of the internet use, including social negative consequences, loss of control of overuse, unsuccessful attempts to reduce using it, the ability to control mood etc. Here are some examples of questions: «How often do you avoid household chores to spend more time online?», "How often do you lose sleep because you are online all night long?", «How often are your grades way down because of the time spent on the Internet?», "How often do you spend online more time as it planned?», "How often do you try to reduce being online and fail by stopping use it?», "How often do you feel Internet addiction or dream like being online right now?" and other questions. Participants are informed aboutparagraphs all referred to their general use of the Internet, including the full range of such activities. The test has good psychometric properties and confidence level [1].

\subsection{Internet Disorder Scale (IDS-15)}

IDS-15 includes the following categories:

-4 items - (escapism and dysfunctional emotional coping (EDEC));

- 4 items - (withdrawal symptoms (WS));

- 4 items - (impairments and dysfunctional self-regulation (IDSR));

- 3 items - (dysfunctional Internet-related self-control (DISC).

Results are evaluated with 5-point Likert scale ranging from 1 (strongly disagree) to 5 (totally agree). A higher number of points indicate an increased likelihood of virtual addiction (Internet addiction) [5, p. 665-675]. 


\subsection{Internet Gaming Disorder Scale - Short Form (IGDS9-SF)}

Test shows gambling addiction using nine positions based on the DSM-5 criteria, where the results are also evaluated with 5-point Likert scale. The Persian version of IGDS9-SF has satisfying internal consistency $(\alpha=.90)$, Test validity, etc. [5, p. 665-675].

\subsection{Bergen Social Media Addiction Scale (BSMAS)}

Test by Griffiths (2005) consists of six elements, including six basic elements of addiction (significance, tolerance, mood change, feedback, conflict, relapse). All criteria are evaluated on a 5-point Likert scale in the range from 1 (very rarely) to 5 (very often). BSMAS assessment of more than 19 out of 30 shows problems of using social networks [5, p.665-675].

\subsection{Cybersex Motives Questionnaire (CysexMQ)}

Test identifies reasons in search of a cyber-sexual experience, such as the desire to "get to know someone", "to have fun", "for masturbation", "for observation", etc. [3, p.601-609].

\subsection{Functional magnetic resonance imaging (fMRI)) and event-related potentials (ERPs)}

Efficient methods for diagnosing can be magnetic resonance imaging (MRI), because in this way the study of neuronal changes in various parts of the brain is carried out. This method is really powerful, it gives accurate results. There is activation in neuronal substrates during the test. Scientists have noticed that, in some cases, it is possible to detect a change in the amplitude of the signals in the brain upon examination images related to the subject of addiction [2].

A positive factor is that magnetic resonance imaging relates to non-invasive methods for studying the neurological processes of mental disorders. Functional imaging methods can help research that relate to the neural features of the formation and progression of virtual addiction, as well as increasing the statistical reliability of clinical data. Changes in the brain signal are analyzed in terms of functional fluctuations (flashes), compared to the baselines [10].

This is a very important type of diagnosis, because the pathogenesis of virtual addiction is not well researched today. Researchers try to receive more 
information about encephalopathy regarding the above-mentioned disorder among adolescents. Virtual addiction can cause changes in socio-behavioural characteristics like chemical addictions. Adolescence is characterized by significant changes in the neurobiological systems in decision-making positions. This is one of the reasons why the younger age group of people is more prone to affective disorders and the emergence of various addictions. Test was done (33 persons participated). The test group consisted of 17 adolescents with an Internet addiction of more than 2 years, namely, 15 men and 2 women, as well as a control group of 16 healthy people, which included 14 men and 2 women (Internet usage $1.3 \pm 0.6$ per hours / day or $4.6 \pm 2.1$ per days / week). It was determined that addicted teens spent their time approximately $4.8 \pm 2.2$ hours / day on the Internet and online games. Participants spent about $6.5 \pm 1.5$ days per week. It was used a 20 -point questionnaire developed by Dr. Kimberly Young for an establishment of the diagnosis, participants underwent a simple medical examination, etc. It was important to exclude accompanying mental disorders, toxicomania or other types of addiction, physical pathologies, pregnancy or the menstrual period in order to get more precise results. People with Internet addiction had a difference in the area of the right anterior cingulate gyrus (limbic area), the right middle cingulate gyrus (limbic area), the left inferior parietal lobe (parietal area) and the left half of the thalamus (subcortical area) [17, e107306].

\subsection{Dual-process models of addiction and he implicit association test}

There is an automatic (implicit) system component that includes memory associations and a controlled (explicit) system under constant self-examination, including cognition, as well as deliberate decisions. By the formation of addiction, the controlled system can be replaced by an automatic system, so people continue to abuse virtual space, despite understanding the negative consequences of this activity. Automated processes are able to make a contribution to the development and maintaining addictions. Understanding of the process of implicit cognition can help in anticipation of using psychoactive substances [9, p. 79-87].

Important method for diagnosing virtual addiction is to study the patient's cognitive processes, namely the processes of perception, understanding, memory, reasoning, etc. Implicit cognition is a predictor of mental disorders such as alcohol and tobacco addiction. A worthwhile method of studying virtual addiction is an implicit association test. Methods had 
demonstrated their effectiveness for evaluating implicit associations by using tobacco, alcohol, marijuana, cocaine and helped to differentiate consumers of these substances from healthy individuals (participants were 15-18 years old). Among people who are addicted to Internet games, there is a positive motivational response to screenshots of online games. For testing to identify virtual addiction, there are 6 Internet-related images (used the WeChat icon, KingofGlory online games, Taobao icon, GoogleChrome icon, Internet Explorer icon and Tencent QQ icon) and 6 mammals (dog, monkey, horse, pig, sheep and dolphin), similarly 6 positive ("happiness", "attractiveness", "relaxation", "excitement", "friendliness" and "public") and 6 neutral words ("general", "calm", "impartial", "brown", "static"and" objective"). Exposure takes approximately 2 seconds [2; 8, p. 308-330].

The questionnaire can be used to determine the addiction specifically on smartphones based on the identification of implicit interactions between positive emotions and images from Internet games. It was used images related to online games and furniture, as well as attribute positive and negative concepts to identify the link between "game-positive" and "furniture-negative". Each of the categories had eight images. The proposed method can be very promising, because addiction to virtual space develops within visual stimulus [9, p. 79-87].

There are common characteristics between addiction to cybersex and chemicals, therefore, the results of the study can establish liaison with implicit associations between pornographic images and positive emotions, which were similar to the data on addiction to chemicals [12, p. 7-12].

\subsection{Diagnostic criteria of Tao and co-authors}

Tao and co-authors developed their diagnostic criteria in order to find out the Internet addiction based on the clinical characteristics of a large group of Chinese patients who thought they had this disorder. Patients with bipolar disorder and / or psychotic disorders took part. As a result of the work carried out, the researchers received the following set of criteria:

- symptomatic criteria, what means symptoms of immersion and abstraction (both must be present);

- one or more of these criteria: tolerance, constant desire and / or unsuccessful attempt to control usage, long-term use, despite problems, loss of other interests, use of the Internet to prevent or alleviate dysphoric mood; 
- criteria of clinically significant deficiency: functional deficiency (loss of work and social productivity), including the loss of significant relationship, job, educational or career opportunities;

- the duration of the disorder should be more than three months and at least six hours of using the Internet per day, not take into account the work and study [16, p. 203-213].

\subsection{Davis cognitive-behavioral model}

According to the 2001 Davis cognitive-behavioral model, there is generalized pathological Internet use (GIA) and specific pathological Internet use (SIA). GIA is a multifactor excessive use of the Internet, which is often accompanied by the loss of time and indirect use of online resources (for example, using social communications through social networking sites, subjects can overuse several different Internet resources, that is, play games, watch pornography, read blogs, surf on informational and / or trading sites, post selfies etc.). Such individuals don't have problematic behavior by lack of access to online or offline pages. SIA sufferers have problematic behavior by lack of access to Internet. Among them there are psychopathological diseases. A characteristic feature is also a tendency to neuroticism, vulnerability to stress, low self-esteem, shyness [1].

\subsection{Griffiths components model}

The model is also known as the "component model," according to which addictions consist of six clear and common components (that is, they consist of significance, mood changes, tolerance, abstraction, conflict and relapse). According to Griffiths, addiction is excessive behavior that shares the key elements of the biopsycho-social process. Based on this model, many questionnaires have been developed, including for the diagnosis of work and game addiction [16, 203-213].

\section{Discussion of the problem}

Modern technologies may be considerably helpful for people in everyday life.

Internet resources and media technology, such as a personal computer, laptop, tablet, smartphone, etc. let users find quickly and efficiently the necessary information, make purchases, study and work. The above-mentioned 
equipment and websites could contribute to the development of hobbies and make spending leisure time more wisely. Because of different possibilities of virtual space, there is a risk of loss of control and the development of addiction. Unfortunately, virtual addiction can develop for various reasons. Such reasons as depression, post-traumatic stress disorder, neurotic disorders, etc., where

Risk factors

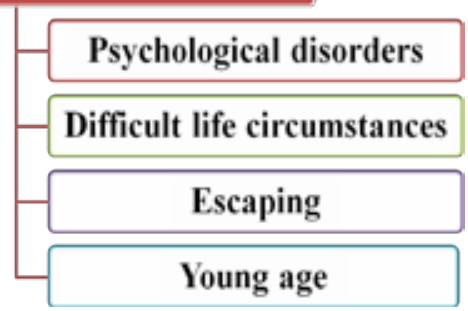

Figure 4. Risk factors escaping plays an important role, that is, a distracting factor from significant life problems. A special risk group includes adolescence due to the physiological and psychological characteristics of this age category (see Figure 4).

For young people, the formation of virtual addiction may deal a heavy blow to both the psycho-physical state and socio-financial stability. People who excessively / pathologically use the Internet and media technology may lose their desire to study and work, as well as the desire to communicate outside the network, which threatens normal everyday life. Today, the possibility of high-quality and timely diagnosis of virtual addiction both by adolescents and by people of other age categories is acquiring an ever-increasing importance. Concomitant psychological disorders complicate the task of differentiating the diagnosis. This must be taken into account during the survey. In addition to age factors, doctors and medical psychologists should take into consideration such factors as gender, social status, some cognitive personal characteristics, etc. The need for diverse approaches to the problem was one of the reasons for the emergence of a large number of different diagnostic questionnaires, as well as the introduction of instrumental methods of study, which include magnetic resonance imaging. The free access to the Internet and media technology for adolescents can be extremely dangerous, there are threats of psychological and physical violence, intimidation, financial difficulties, suicidal behavior, Internet fraud, etc. Parents should have meaningful conversations with their children, be able to shorten the online period and effectively block access to potentially dangerous resources. These preventative actions, according to the recommendations of many modern scientists, can help prevent many serious consequences for a teenager (see Figure 5). 


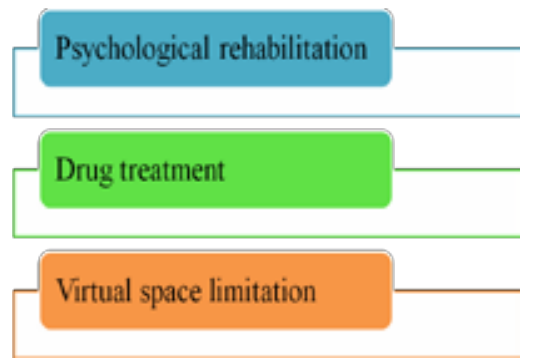

Figure 5. The main methods of treatment

\section{Conclusions}

Virtual addiction may be developed for various reasons. It is necessary to use a multifactorial approach to the problem of diagnosing virtual dependence. The doctor and medical psychologist should choose the type of questionnaire according to the patient's characteristics and take into account his age, gender, social status, physical and psychological disorders. Because of the lack of effective solution of everyday problems by teenagers, it is possible to develop various psycho-emotional disorders, chemical addictions, and falling under the influence of destructive organizations of a political and religious nature. An important role in the prevention of virtual addiction plays healthy psycho-social environment, understanding and loving parents / friends who can support, give the right advice and help by life's difficulties. Such support significantly reduces the need for escaping. Further research of virtual addiction is required to effectively diagnose and treat this disorder. Improving the quality of research, creating a strong scientific base, and improving the legislative regulation of this problem can significantly contribute to reducing risks that appear by the pathological use of Internet resources and media technology.

\section{References:}

1. Brand, M., Laier, C., \& Young, K. (2014). Internet addiction: coping styles, expectancies, and treatment implications. Frontiers in Psychology, 5. doi: 10.3389/fpsyg.2014.01256

2. Chen, L., Zhou, H., Gu, Y., Wang, S., Wang, J., \& Tian, L. et al. (2018). The Neural Correlates of Implicit Cognitive Bias Toward Internet-Related Cues in Internet Addiction: An ERP Study. Frontiers in Psychiatry, 9. doi: 10.3389/fpsyt.2018.00421

3. Franc, E., Khazaal, Y., Jasiowka, K., Lepers, T., Bianchi-Demicheli, F. and Rothen, S. (2018). Factor structure of the Cybersex Motives Questionnaire. Journal of Behavioral Addictions, 7(3), pp. 601-609. 
4. Kawabe, K., Horiuchi, F., Ochi, M., Oka, Y., \& Ueno, S. (2016). Internet addiction: Prevalence and relation with mental states in adolescents. Psychiatry and Clinical Neurosciences, 70(9), 405-412. doi: 10.1111/pcn.12402

5. Lin, C., Ganji, M., Pontes, H., Imani, V., Broström, A., Griffiths, M. and Pakpour, A. (2018). Psychometric evaluation of the Persian Internet Disorder Scale among adolescents. Journal of Behavioral Addictions, 7(3), pp. 665-675.

6. Love, T., Laier, C., Brand, M., Hatch, L., \& Hajela, R. (2015). Neuroscience of Internet Pornography Addiction: A Review and Update. Behavioral Sciences, 5(3), 388-433. doi: 10.3390/bs5030388

7. Mihajlov, M., \& Vejmelka, L. (2017). INTERNET ADDICTION: A REVIEW OF THE FIRST TWENTY YEARS. Psychiatria Danubina, 29(3), 260-272. doi: $10.24869 /$ psyd.2017.260

8. Rae, J., \& Olson, K. (2018). Test-retest reliability and predictive validity of the Implicit Association Test in children. Developmental Psychology, 54(2), 308-330. doi: 10.1037/dev0000437

9. Roh, D., Bhang, S., Choi, J., Kweon, Y., Lee, S., \& Potenza, M. (2018). The validation of Implicit Association Test measures for smartphone and Internet addiction in at-risk children and adolescents. Journal of Behavioral Addictions, 7(1), 79-87. doi: 10.1556/2006.7.2018.02

10. Sepede, G., Tavino, M., Santacroce, R., Fiori, F., Salerno, R., \& Di Giannantonio, M. (2016). Functional magnetic resonance imaging of internet addiction in young adults. World Journal of Radiology, 8(2), 210. doi: 10.4329/wjr.av8.i2.210

11. Shek, D., \& Yu, L. (2012). Internet Addiction Phenomenon in Early Adolescents in Hong Kong. The Scientific World Journal, 2012, 1-9. doi: 10.1100/2012/104304

12. Snagowski, J., Wegmann, E., Pekal, J., Laier, C., \& Brand, M. (2015). Implicit associations in cybersex addiction: Adaption of an Implicit Association Test with pornographic pictures. Addictive Behaviors, 49, 7-12. doi: 10.1016/ j.addbeh.2015.05.009

13. Suicide across the world (2016). World Health Organization (2019). Retrieved 31 July 2019, from https://www.who.int/mental_health/prevention/ suicide/suicideprevent/en/

14. Suicide. World Health Organization (2019). Retrieved 31 July 2019, from https://www.who.int/news-room/fact-sheets/detail/suicide

15. Te Wildt, BT. (2018). Diagnosis and Therapy of Internet Addiction. Rehabilitation (Stuttg), 57(05), pp. 321-333.

16. Van Rooij, A., \& Prause, N. (2014). A critical review of "Internet addiction" criteria with suggestions for the future. Journal of Behavioral Addictions, 3(4), 203-213. doi: 10.1556/jba.3.2014.4.1

17. Wee, C., Zhao, Z., Yap, P., Wu, G., Shi, F., \& Price, T. et al. (2014). Disrupted Brain Functional Network in Internet Addiction Disorder: A RestingState Functional Magnetic Resonance Imaging Study. Plos ONE, 9(9), e107306. doi: 10.1371/journal.pone.0107306

18. Zajac, K., Ginley, M., Chang, R., \& Petry, N. (2017). Treatments for Internet gaming disorder and Internet addiction: A systematic review. Psychology of Addictive Behaviors, 31(8), 979-994. doi: 10.1037/adb0000315 